\title{
Breakdown of the Quantum Hall Effect
}

\author{
D.K. Madde ${ }^{a}$, L.B. Rigat ${ }^{a}$, W. Desrat ${ }^{a}$, M. Potemski ${ }^{a}$, \\ J.C. Portal ${ }^{a}$, L. Eaves ${ }^{b}$, Z.R. Wasilewski ${ }^{c}$, A.I. TOropov ${ }^{d}$ \\ AND G. HILL ${ }^{e}$ \\ ${ }^{a}$ Grenoble High Magnetic Field Laboratory, Max Planck Institut \\ Festkörperforschung and Centre National de la Recherche Scientifique \\ BP166, 38042 Grenoble, France \\ ${ }^{b}$ Department of Physics, University of Nottingham, Nottingham, NG7 2RG, U.K. \\ ${ }^{c}$ Institute for Microstructural Sciences, National Research Council \\ Ottawa, K1A 0R6, Canada \\ ${ }^{d}$ Institute of Semiconductor Physics, Novossibirsk 630090, Russia \\ ${ }^{e}$ Department of Electronic and Electrical Engineering, University of Sheffield
} Sheffield S1 4DU, U.K.

\begin{abstract}
The breakdown of the dissipationless conductance in the integer and fractional quantum Hall effect regime is reviewed. The temperature dependence of the critical current and of the critical magnetic field at breakdown bears a striking resemblance to the phase diagram of the phenomenological two-fluid Gorter-Casimir model for superconductivity. In addition, a remarkably simple scaling law exists between different filling factors.
\end{abstract}

PACS numbers: 71.10.Pm, 74.25.Dw

\section{Introduction}

At integer filling factors and under quantum Hall conditions the resistance of a 2-dimensional electron gas (2DEG) is vanishingly small and intuitively the electrical properties resemble those of a superconductor. The analogy with superconductivity is rather naive and not without problems; principally no Meissner effect is observed and the resistivity is exponentially small rather than zero. Nevertheless, some theoretical works exist linking the fractional quantum Hall effect (FQHE) to superconductivity [1-4]. Theory linking the integer quantum Hall effect (IQHE) to superconductivity is more scarce. It has been suggested that the 
backscattering between edge states at breakdown is equivalent to the weak link in a Josephson junction, so that in sufficiently narrow quantum Hall systems it should be possible to observe Josephson type oscillations in the IQHE regime [5,6].

In the quantum Hall regime the "dissipationless" conductance can be destroyed by either applying a sufficiently large current or changing the magnetic field so as to move away from integer filling factor. The breakdown of the IQHE has attracted considerable experimental and theoretical interest [7-17]. While the exact mechanism for the breakdown remains controversial, what is clear is that the breakdown is driven by the largest electric field present in the system, namely the electric field resulting from the Hall voltage $V_{\mathrm{H}}=I h / \nu e^{2}$ for a current $I$ at integer filling factor $\nu$.

In this paper, rather than focusing on the microscopic model, we show that it is possible to map out a phase diagram (critical current or critical magnetic field versus temperature) for the breakdown of the QHE. We first review our earlier work [18] for even integer filling factors where we show that the measured phase diagram bears a striking resemblance to the phase diagram for the coercive field in a superconductor. In addition, the phase diagrams for different filling factors are all related by a remarkably simple scaling law. This work is then extended to include odd and fractional filling factors.

The samples investigated here are modulation doped heterojunctions or single quantum wells grown by molecular beam epitaxy. Hall bars were patterned to have a width $d=250 \mu \mathrm{m}$ with $750 \mu \mathrm{m}$ between voltage probes. For the measurements the sample was mounted in variable temperature insert or directly in the mixing chamber of a dilution refrigerator. The magnetic field was generated using either a superconducting magnet $(0-15 \mathrm{~T})$ or one of the $20 \mathrm{MW}$ resistive magnets $(0-28 \mathrm{~T})$ at the Grenoble High Magnetic Field Laboratory.

\section{Even filling factors}

In order to investigate even filling factors an $8.2 \mathrm{~nm}$ quantum well sample with a mobility of $11 \mathrm{~m}^{2} \mathrm{~V}^{-1} \mathrm{~s}^{-1}$ and carrier density of $7.3 \times 10^{11} \mathrm{~cm}^{-2}$ was used. The high carrier density gives access to a large number of even filling factors. A typical magnetoresistance trace measured at $2.0 \mathrm{~K}$ is shown in Fig. 1a. The critical current $I_{\mathrm{c}}$ was determined by measuring four terminal $I-V$ characteristics for magnetic fields at $10 \mathrm{mT}$ intervals in such a way as to sweep through the particular filling factor [18]. A plot of $I_{\mathrm{c}}$ versus magnetic field gives a slightly asymmetric Gaussian (Fig. 1b) with the maximum corresponding to the magnetic field for which $\nu$ is integer. The critical current for a given filling factor is taken to be the maximum of the Gaussian.

The temperature dependence of $I_{c}$ is shown in Fig. 2a. For a given filling factor $I_{c}$ has an almost constant value at low temperatures before decreasing and then vanishing at a critical temperature $T_{c}$. The temperature dependence of $I_{c}$ is 


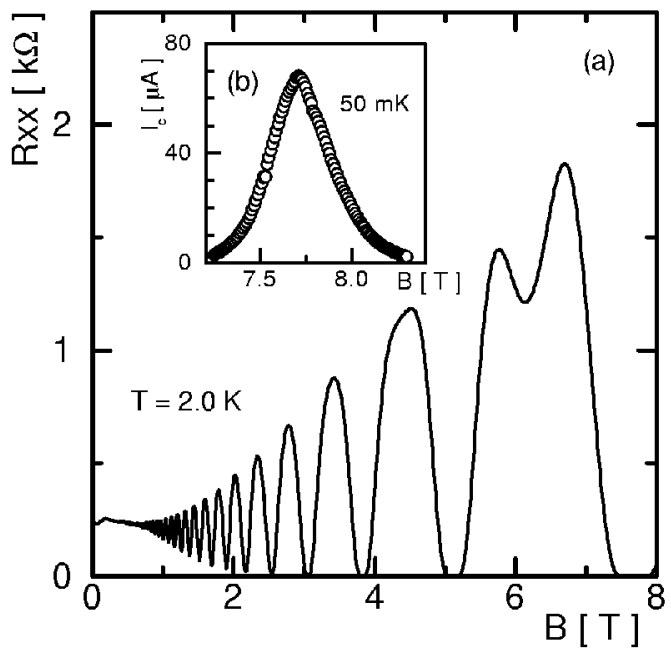

Fig. 1. (a) Magnetoresistance measured at $T=2.0 \mathrm{~K}$ for the $8.2 \mathrm{~nm}$ QW sample. (b) Dependence of the critical current on magnetic field in the vicinity of $\nu=4$.

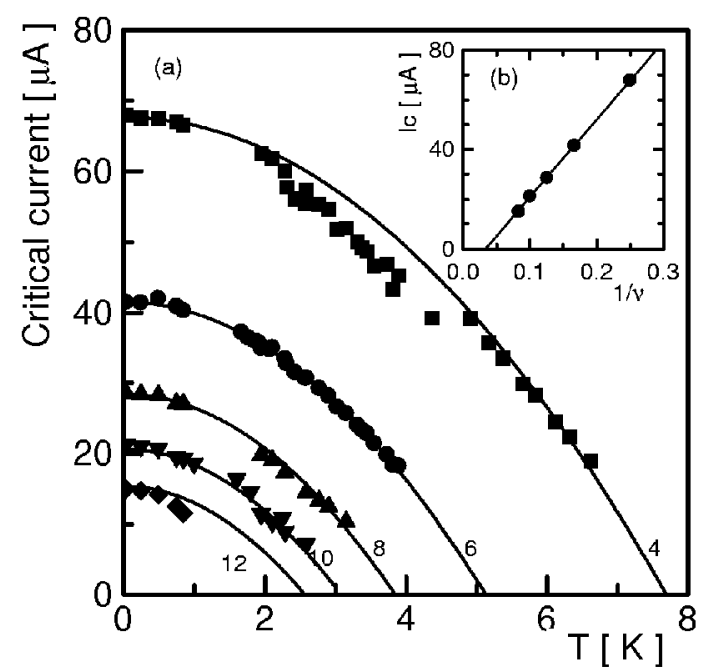

Fig. 2. (a) Critical current $\left(I_{\mathrm{c}}\right)$ as a function of temperature for even filling factors for the $8.2 \mathrm{~nm}$ QW sample. The solid lines are calculated using Eq. (1) as described in the text. (b) $I_{c}(40 \mathrm{mK})$ versus inverse filling factor $(1 / \nu)$. The solid line is a least squares fit to the data.

remarkably similar to the phase diagram for the coercive field of a superconductor (for superconductors $I_{\mathrm{C}}$ and $H_{\mathrm{C}}$ are equivalent since the superconductivity is also quenched when the current is sufficient to produce its own critical magnetic field). The solid lines are generated using an expression similar to that for the 
phenomenological Gorter-Casimir [19] two-fluid model for superconductivity

$$
I_{\mathrm{c}}(T, \nu)=\left(\frac{I_{\mathrm{c} 0}}{\nu}-\frac{I_{\mathrm{c} 0}}{\nu_{0}}\right)\left[1-\left(\frac{T}{T_{\mathrm{C} 0} / \nu}\right)^{2}\right],
$$

where $I_{\mathrm{c} 0}=314 \mu \mathrm{A}$ and $\nu_{0}=30$ are obtained from the straight line fit to $I_{\mathrm{c}}(T \approx 0)$ versus $1 / \nu$ shown in Fig. $2 \mathrm{~b}$. The value of $\nu_{0}$ has the physical significance that this is the largest filling factor for which the conduction is expected to be dissipationless at zero temperature. From the magnetic field at which Shubnikov-de Haas oscillations are first observed this corresponds to the condition $\omega_{\mathrm{c}} \tau \approx 2$. The only remaining parameter $T_{c 0}=31 \mathrm{~K}$ is found by fitting Eq. (1) to the temperature dependence of $I_{c}$ for the filling factor $\nu=6$. The temperature dependence of $I_{c}$ can then be generated using Eq. (1) for the other filling factors with no adjustable parameters. The agreement between the data and the model for all filling factors is remarkable and an equally good agreement has been found in all samples. We note that the critical current and the critical temperature scale as the cyclotron energy $\left(\hbar \omega_{\mathrm{c}} \propto 1 / \nu\right)$.

It is interesting to compare the observed scaling with the predictions of the quasi-elastic inter Landau level scattering (QUILLS) model for the breakdown [14]. Experimentally, the critical Hall electric field at $T=0$,

$$
F_{\mathrm{C}}=V_{\mathrm{H}} / d \approx \frac{I_{0}}{\nu} \frac{h / e^{2}}{\nu} \frac{1}{d} \propto \frac{1}{\nu^{2}} .
$$

In the QUILLS model breakdown occurs when quasi-elastic scattering to lowest unoccupied Landau level can occur. The critical Hall electric field is estimated using the classical turning points of the simple harmonic oscillator states to be

$$
F_{\mathrm{C}}=\frac{\hbar \omega_{\mathrm{C}}}{e \ell_{B}\left[(\nu-1 / 2)^{1 / 2}+(\nu+1 / 2)^{1 / 2}\right]},
$$

where $\hbar \omega_{\mathrm{c}} \propto 1 / \nu$ is the cyclotron energy and $\ell_{B}=(\hbar / e B)^{1 / 2} \propto \nu^{1 / 2}$ is the magnetic length. The predicted scaling

$$
F_{\mathrm{C}} \propto \frac{1}{\nu^{3 / 2}\left[(\nu-1 / 2)^{1 / 2}+(\nu+1 / 2)^{1 / 2}\right]}
$$

agrees with the observed scaling $\approx \frac{1}{\nu^{2}}$ to within $1 \%$ for the filling factors investigated.

We now turn our attention to the temperature dependence of the width of the dissipationless regions in the magnetoresistance traces. Pursuing the analogy with the Gorter-Casimir model, we will try to map out a $B_{c}$ versus $T$ phase diagram for the different filling factors. Magnetoresistance traces were measured at different temperatures and with a small current $\approx 10-100 \mathrm{nA}$ using low frequency $(10.7 \mathrm{~Hz})$ phase sensitive detection. $B_{c}$ is defined as the half width of the dissipationless region at each filling factor. Such a phase diagram for even integer filling factors is shown in Fig. 3a. At first sight the data points bear little resemblance to the 
Gorter-Casimir phase diagram. However, as indicated by the solid and dashed lines, it is possible to decompose this phase diagram into a low temperature (LT) and high temperature (HT) phase. The HT phase can be fitted using

$$
B_{\mathrm{C}}(T, \nu)=\left(\frac{B_{\mathrm{c} 0}^{\mathrm{HT}}}{\nu^{2}}-\frac{B_{\mathrm{c} 0}^{\mathrm{HT}}}{\nu_{0}^{2}}\right)\left[1-\left(\frac{T}{T_{\mathrm{c} 0}^{\mathrm{HT}} / \nu}\right)^{2}\right],
$$

where $\nu_{0}=30$ (as before) and $B_{\mathrm{c} 0}^{\mathrm{HT}}=6.7 \mathrm{~T}$ and $T_{\mathrm{c} 0}^{\mathrm{HT}}=54 \mathrm{~K}$ are determined by fitting to the data for $\nu=8$. The curves for all the other filling factors (dashed lines in Fig. 3a) are then generated using Eq. (2) with no adjustable parameters. For $\nu=8,10,12$ the agreement is good while for lower filling factors there is a deviation with the data dipping below the predicted curve for a substantial part of the phase diagram.

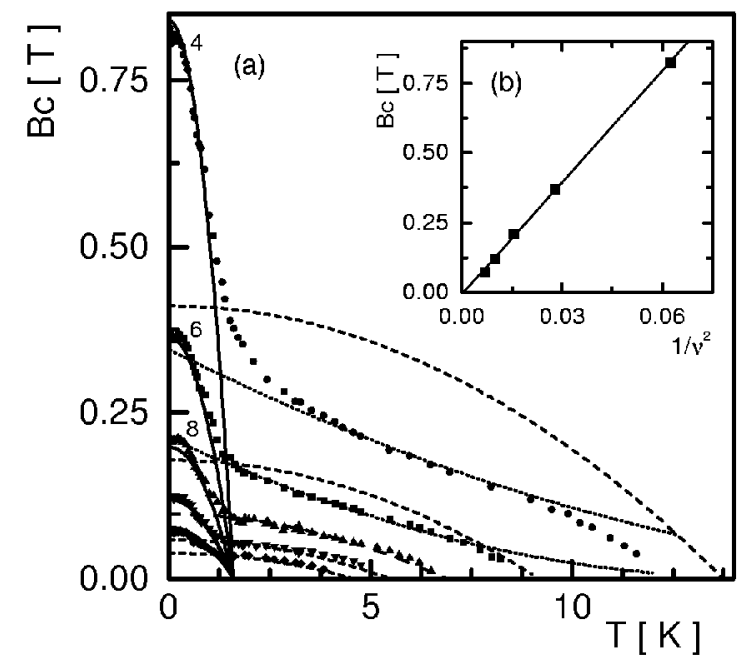

Fig. 3. (a) Critical magnetic field $\left(B_{\mathrm{c}}\right)$ versus temperature for the $8.2 \mathrm{~nm}$ QW sample. Even filling factors $(\nu=4,6,8,10,12)$ are shown and reveal the existence of two phases. The dashed (HT phase), solid (LT phase), and dotted (melting) lines are calculated using Eqs. (2)-(4), respectively. (b) $B_{c}(40 \mathrm{mK})$ versus $1 / \nu^{2}$. The solid line is a least squares fit to the data.

The LT phase in Fig. 3a can be fitted using

$$
B_{\mathrm{c}}(T, \nu)=\left(\frac{B_{\mathrm{c} 0}^{\mathrm{LT}}}{\nu^{2}}-\frac{B_{\mathrm{c} 0}^{\mathrm{LT}}}{\nu_{0}^{2}}\right)\left[1-\left(\frac{T}{T_{\mathrm{c} 0}^{\mathrm{LT}}}\right)^{2}\right],
$$

which is identical to Eq. (2) except that $T_{c}=T_{\mathrm{c} 0}^{\mathrm{LT}}$ no longer scales as the cyclotron energy i.e. in the LT phase the critical temperature is the same for all filling factors. In contrast to the behaviour of $I_{c}(T \approx 0)$, for both the LT and HT phases, $B_{c}(T \approx 0)$ scales as the cyclotron energy squared $\left(1 / \nu^{2}\right)$ as shown in Fig. 3b. From 
the slope $B_{\mathrm{C} 0}^{\mathrm{LT}}=13.4 \mathrm{~T}$. The solid lines in Fig. 3a are generated by fitting to the low temperature $\nu=4$ data to determine $T_{\mathrm{c} 0}^{\mathrm{LT}}=1.6 \mathrm{~K}$ with $\nu_{0}=30$ as before.

The critical magnetic field $B_{c}$ is determined simply by the number of localised states in the relevant Landau level (LL). It is easy to show that the normalised density of localised states in the LL $n_{\mathrm{loc}} / n_{\mathrm{LL}}=2 \nu B_{\mathrm{c}} / B_{\mathrm{F}}(\approx 20 \%$ for $\nu=4$ at $T \approx 0$ with the fundamental field $\left.B_{\mathrm{F}}=30.3 \mathrm{~T}\right)$. As $B_{\mathrm{c}} \propto 1 / \nu^{2}$ this implies that the density of localised states within a LL is proportional to the magnetic field $(\propto 1 / \nu)$. The critical current which is determined with the Fermi level centred in the localised states between LLs appears to be insensitive to the exact number of delocalised states since only one phase is observed. It is not clear why two phases are observed for $B_{\mathrm{c}}$ but this must be linked to increased localisation at low temperatures. In the usual scaling theory picture of the IQHE this increased localisation is interpreted as being due the quantum interference of coherent electrons $[20,21]$. The electrons are considered to be localised when the localisation length $\xi(E) \sim\left|E-E^{*}\right|^{-\mu}$ is larger than the effective sample size which in general is given by the phase coherence length $L_{\phi} \sim T^{-p / 2}$. Here $E^{*}$ is the energy at the centre of the Landau level, $\mu \approx 2.34$ is universal and $p=1$ in two dimensions. Using scaling theory a reasonable fit (not shown) to the low temperature phase in Fig. 3 a can be obtained further supporting the hypothesis coherence is responsible for the observed increased localisation at low temperatures.

We noted above that for lower filling factors in Fig. 3 the data dips below the high temperature fitting curve for a substantial part of the phase diagram. A similar deviation is observed in the phase diagram of high temperature $\left(\mathrm{HT}_{\mathrm{c}}\right)$ superconductors and is associated with the melting curve of the Abrikosov vortex lattice [22]. In the liquid phase, when a current is applied, the vortices are free to move under the influence of the Lorentz force which leads to dissipation and the superconductivity is quenched. In type II superconductors the critical field can be enhanced by the addition of impurities which pin the vortices and prevent flux jumping while in the QHE disorder increases the number of localised states and hence enhances $B_{c}$. For the IQHE the "melting" would correspond to a delocalisation of cyclotron orbit centres. It is therefore natural to make an analogy with type II superconductors and in particular HT $_{c}$ superconductors with weak interlayer coupling which show a $2 \mathrm{D}$ behaviour. The melting behaviour suggested by the deviation of the data points from Eq. (2) can be fitted to the functional form of the Abrikosov lattice melting curve

$$
B_{\mathrm{m}}(T, \nu)=\left(\frac{B_{\mathrm{m}}}{\nu}-\frac{B_{\mathrm{m}}}{\nu_{0}}\right)\left(1-\frac{T}{T_{\mathrm{m}} / \nu}\right)^{2} .
$$

A good fit for the lower $\nu$ values can be obtained with $B_{\mathrm{m}}=1.6 \mathrm{~T}, \nu_{0}=30$ and $T_{\mathrm{m}}=90 \mathrm{~K}$. Here the $1 / \nu$ scaling law has been determined empirically. The predicted melting curves for $\nu=8,10,12$ (not shown) lie above the usual phase boundary and hence these filling factors are unaffected. For $\nu=6$, the data follows the melting curve to join the HT phase near $T_{c}$ while for $\nu=4$, 
the data dips below the melting curve close to the predicted $T_{c}$ and appears to have a critical temperature $\approx 2 \mathrm{~K}$ lower than expected. This is similar to the Berezinskii-Kosterlitz-Thouless (BKT) behaviour observed in 2D superconductors [23-25] due to intrinsic vortex-antivortex excitations which destroy the topological order and lead to a critical temperature $T_{\mathrm{BKT}}$ which is typically $\approx 1 \mathrm{~K}$ smaller than $T_{\mathrm{c}}$. Although the comparison of the $\mathrm{HT}_{\mathrm{c}}$ and QHE phase diagrams is clearly intriguing such an interpretation of our data would nevertheless be highly speculative.

\section{Odd filling factors}

A systematic investigation of odd filling factors in the $8.2 \mathrm{~nm}$ quantum well sample is not possible due to the limited number of odd filling factors which are dissipationless and in the available magnetic field range. For this reason a heterojunction sample (carrier density $1.3 \times 10^{11} \mathrm{~cm}^{-2}$ and mobility $50 \mathrm{~m}^{2} \mathrm{~V}^{-1} \mathrm{~s}^{-1}$ ) was chosen to investigate odd filling factors. The number of filling factors which are dissipationless $(\nu=1,3,5)$ is limited due to the critical collapse of spin splitting $[26,27]$. The scaling for odd filling factors is complicated by the many-body nature of the spin gap which is in general dominated by the exchange energy [28, 29]. We demonstrate that the data for odd filling factors can be fitted reasonably well with the phenomenological model developed for the even filling factors provided this is modified to take into account the many-body nature of the gap.

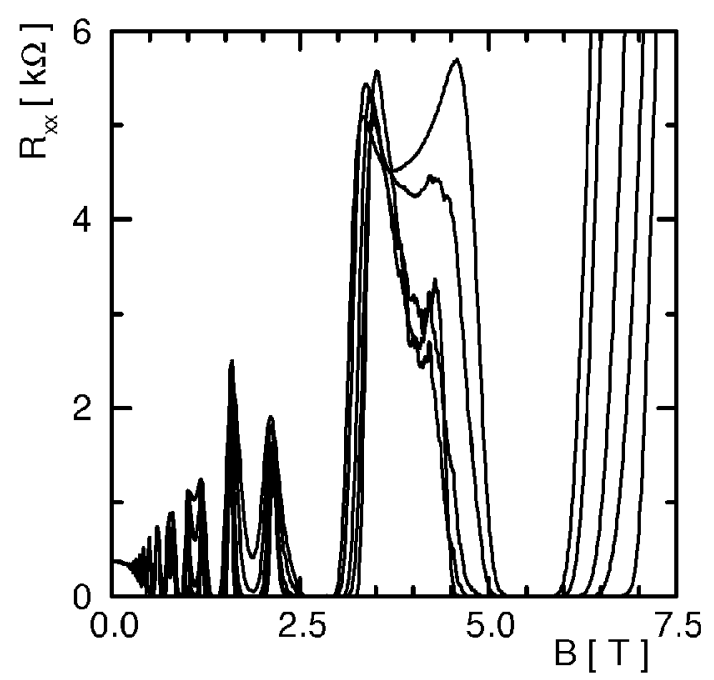

Fig. 4. Typical magnetoresistance traces for the $n=1.3 \times 10^{11} \mathrm{~cm}^{-2}$ heterojunction sample measured at temperatures $0.05-1.1 \mathrm{~K}$.

Typical magnetoresistance traces measured for temperatures between $50 \mathrm{mK}$ and $1.1 \mathrm{~K}$ are shown in Fig. 4. The phase diagrams for the breakdown are deter- 
mined as for the even filling factors. The temperature dependence of the critical current, $I_{c}$, is shown in Fig. 5a for filling factors $\nu=1,3,5$. For a given filling factor $I_{\mathrm{C}}$ has a constant value at low temperatures before decreasing rapidly and then vanishing at a critical temperature $T_{\mathrm{c}}$. In contrast to the case for even filling factors it is not possible to fit this dependence with the phenomenological Gorter-Casimir [19] two-fluid model for superconductivity. Instead a modified form with a $T^{4}$ power dependence is required to correctly fit the rapid decrease as $T_{\mathrm{C}}$ is approached

$$
I_{\mathrm{C}}(T, \nu)=\left(\frac{I_{\mathrm{c} 0}}{\sqrt{\nu}}-\frac{I_{\mathrm{c} 0}}{\sqrt{\nu_{0}}}\right)\left[1-\left(\frac{T}{T_{\mathrm{c} 0} / \sqrt{\nu}}\right)^{4}\right],
$$

where $I_{\mathrm{c} 0}=49.2 \mu \mathrm{A}$ and $\nu_{0}=5.1$ are obtained from the straight line fit to $I_{\mathrm{C}}(T \approx 0)$ versus $1 / \sqrt{\nu}$ shown in Fig. $5 \mathrm{~b}$. As for the even filling factors the value of $\nu_{0}$ has the physical significance that filling factors larger than $\nu_{0}$ are not expected to be dissipationless at zero temperature. The scaling $(1 / \sqrt{\nu})$ between different filling factors in Eq. (5) has also been modified to take into account the many-body nature of the spin gap. For even filling factors the critical current and critical temperature scales as the cyclotron gap, i.e. $1 / \nu$. Assuming that the spin gap is dominated by the exchange energy the spin gap should scale roughly as the Coulomb energy $e^{2} / \varepsilon \ell_{B} \propto 1 / \sqrt{\nu}$ (here $\ell_{B}=\sqrt{\hbar / e B}$ is the magnetic length). The remaining parameter $T_{\mathrm{c} 0}=1.43 \mathrm{~K}$ is found by fitting $\mathrm{Eq}$. (5) to the temperature dependence of $I_{\mathrm{c}}$ for the filling factor $\nu=1$. The temperature dependence of $I_{\mathrm{c}}$ can then be generated using Eq. (5) for the other filling factors with no adjustable

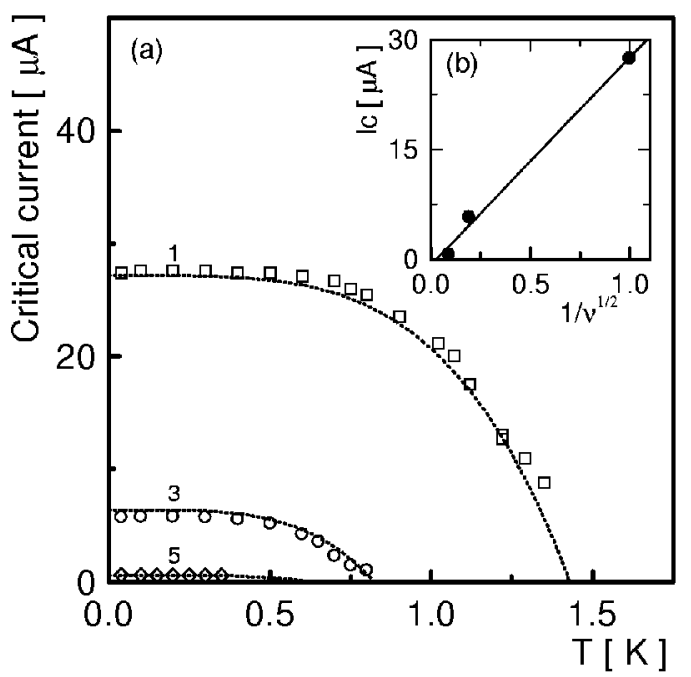

Fig. 5. (a) Critical current $I_{\mathrm{c}}$ as a function of temperature for the $n=1.3 \times 10^{11} \mathrm{~cm}^{-2}$ heterojunction sample. The dissipationless odd filling factors $\nu=1,3,5$ are shown. The dotted lines are fits generated using Eq. (5) as described in the text. (b) $I_{\mathrm{c}}(T \approx 0)$ versus $1 / \sqrt{\nu}$, the solid line is a least squares fit to the data points. 
parameters (dotted lines in Fig. 5a). The agreement between the data and the model for all filling factors is reasonably good. While it is possible to obtain a reasonable fit to $I_{\mathrm{c}}(T \approx 0)$ assuming a $1 / \nu$ dependence of the gap the critical temperature can only be fitted by assuming a $1 / \sqrt{\nu}$ behaviour.

The reason for the $T^{4}$ dependence is less evident. In the QUILLS model [14], the breakdown of the IQHE breakdown occurs when the Hall voltage is sufficiently large to align states of the full and empty Landau levels in the vicinity of the Fermi energy over a spatial distance for which there is significant overlap of the wave functions. Breakdown then occurs via quasi-elastic scattering processes between the Landau levels. The Hall voltage is proportional to the current and thus the critical current is expected to scale as the gap. This suggests that the rapid decrease in $I_{\mathrm{c}}$ ( $T^{4}$ dependence) might be due to a collapse of the exchange enhanced spin gap with increasing temperature. While this interpretation is speculative, we stress that all samples studied show the same $T^{4}$ behaviour for the critical current at odd filling factors.

We now turn our attention to the temperature dependence of the width of the dissipationless regions in the magnetoresistance traces. The measurements (Fig. 4) were performed with a small current $\approx 10-100 \mathrm{nA}$ using low frequency $(10.7 \mathrm{~Hz})$ phase sensitive detection. The temperature dependence of $B_{\mathrm{c}}$ for odd integer filling factors is shown in Fig. 6a. The phase diagram is very similar to that for even filling factors. Notably, two phases are observed: a HT phase with a

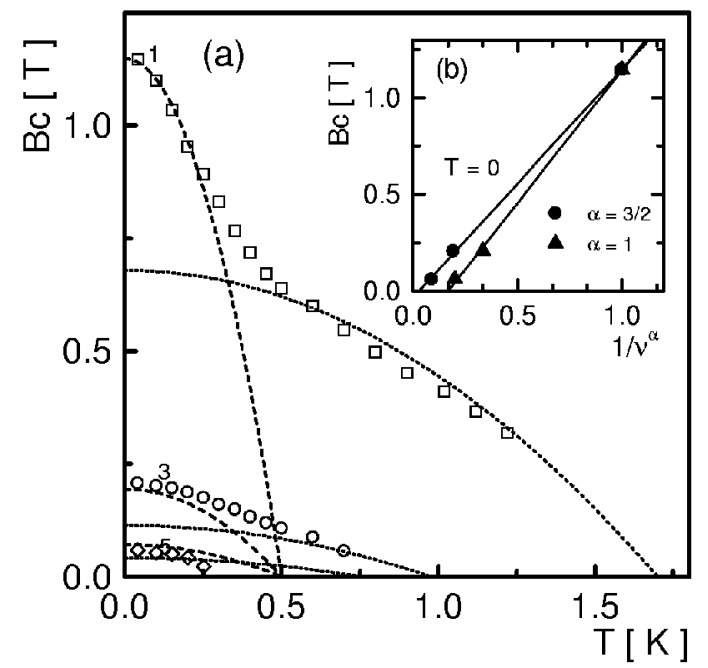

Fig. 6. (a) Critical magnetic field $B_{\mathrm{c}}$ as a function of temperature for the $n=$ $1.3 \times 10^{11} \mathrm{~cm}^{-2}$ heterojunction sample. The dotted and dashed lines are fits generated using Eq. (6) and Eq. ( 7 ) as described in the text. (b) $B_{c}(T \approx 0)$ versus $1 / \nu^{\alpha}$, the solid lines are a least squares fit to the data points plotted with $\alpha=3 / 2$ and $\alpha=1$. 
critical temperature which scales as $1 / \sqrt{\nu}$ and a LT phase which by analogy with even filling factors is expected to have a $T_{c}$ which is independent of filling factor. In contrast to the critical current the temperature dependence is well fitted by a conventional $\left[\left(1-\left(T / T_{\mathrm{C}}\right)^{2}\right]\right.$ Gorter-Casimir phenomenological model (dotted and dashed lines in Fig. 6a).

$B_{c}$ is a direct measure of the number of localised states $\left(n_{\mathrm{loc}}=2 \nu B_{\mathrm{c}} / \Phi_{0}\right)$ between spin Landau levels which should be independent of the gap in the high field limit in which spin Landau level mixing can be neglected. It is not possible to unambiguously determine the correct scaling of $B_{\mathrm{c}}(T \approx 0)$ as can be seen in Fig. $6 \mathrm{~b}$ in which $1 / \nu$ and $1 / \nu^{3 / 2}$ dependence give equally good fits. $B_{\mathrm{c}}(T \approx 0) \propto 1 / \nu$ would imply that $n_{\text {loc }}$ was independent of filling factor (or gap). On the other hand, $B_{\mathrm{c}}(T \approx 0) \propto 1 / \nu^{3 / 2}$ would imply that $n_{\text {loc }} \propto 1 / \sqrt{\nu}$ scales as the spin gap which would imply a constant (energy independent) background of localised states. For even filling factors $n_{\text {loc }} \propto 1 / \nu$ can be interpreted as scaling as the cyclotron energy (i.e. a constant background of localised states) or as scaling with the number of the Landau levels (i.e. the localised states at $B=0$ collapse onto $\nu$ Landau levels in a magnetic field). The dotted and dashed lines in Fig. 6 a are generated using

$$
B_{\mathrm{c}}(T, \nu)=\left(\frac{B_{\mathrm{c} 0}^{\mathrm{HT}}}{\nu^{\alpha}}-\frac{B_{\mathrm{c} 0}^{\mathrm{HT}}}{\nu_{0}^{\alpha}}\right)\left[1-\left(\frac{T}{T_{\mathrm{c} 0} / \sqrt{\nu}}\right)^{2}\right]
$$

and

$$
B_{\mathrm{c}}(T, \nu)=\left(\frac{B_{\mathrm{c} 0}^{\mathrm{LT}}}{\nu^{\alpha}}-\frac{B_{\mathrm{c} 0}^{\mathrm{LT}}}{\nu_{0}^{\alpha}}\right)\left[1-\left(\frac{T}{T_{\mathrm{c} 0}^{\mathrm{LT}}}\right)^{2}\right]
$$

for the HT and LT phases, respectively, with $\alpha=3 / 2$ although $\alpha=1$ would give an equally good fit but with slightly different parameters. Clearly, further measurements on high density and high mobility samples in order to have access to a large number of odd filling factors are required in order to determine unambiguously the correct scaling law.

\section{Fractional filling factors}

For the investigation of fractional filling factors a high mobility heterojunction sample (carrier density $1.6 \times 10^{11} \mathrm{~cm}^{-2}$ and mobility $500 \mathrm{~m}^{2} \mathrm{~V}^{-1} \mathrm{~s}^{-1}$ ) was used. Magnetoresistance traces measured for temperatures between $50 \mathrm{mK}$ and $1 \mathrm{~K}$ are shown in Fig. 7. While minima in the resistance are clearly observed for a large number of fractional occupancies only filling factors $1 / 3,2 / 3$, and $2 / 5$ show dissipationless conduction. The gap for fractional filling factors is approximately two orders of magnitude smaller than the cyclotron gap for even filling factors so that the critical current for the fractional states was too small to allow a systematic study.

We therefore concentrated on measuring the phase diagram for the critical magnetic field which is shown in Fig. 8. Once again it is possible to decompose the 


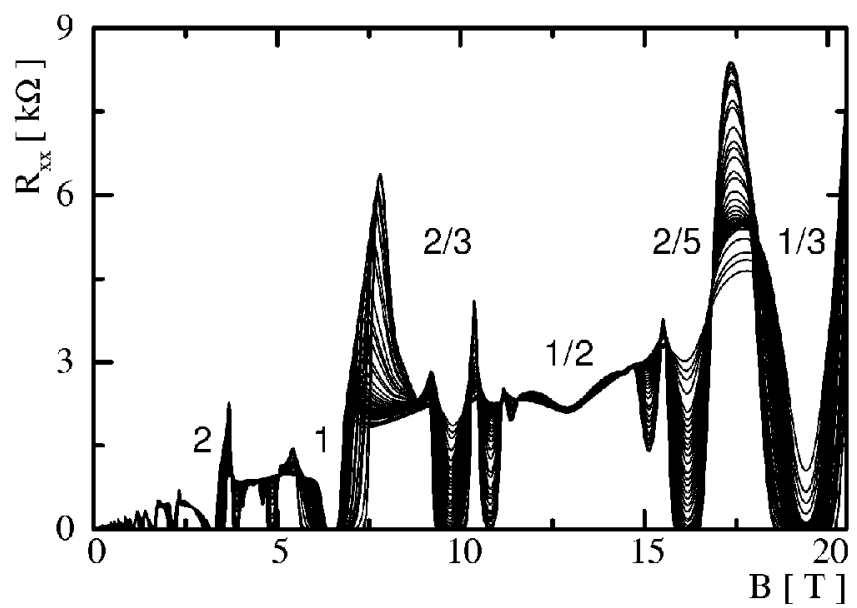

Fig. 7. Typical magnetoresistance traces for the $n=1.6 \times 10^{11} \mathrm{~cm}^{-2}$ heterojunction sample measured at temperatures $0.05-1.0 \mathrm{~K}$.

phase diagram into a low and high temperature phase (as indicated by the dashed and dotted lines) although the transition to the low temperature phase occurs at $\approx 150 \mathrm{mK}$ approximately an order of magnitude smaller than the transition temperature for integer filling factors. Due to the non-trivial dependence of the fractional gaps on filling factor no simple scaling law between different fractional filling factors is expected. From an experimental point of view the limited number of dissipationless fractions does not allow to determine if a simple scaling law exists.

For integer filling factors the low temperature phase can be interpreted using scaling theory in which the increased localisation at low temperatures is a result of the quantum interference of electrons [20, 21]. Such an effect requires phase coherence, which is responsible for a number of well-known effects such as weak localisation, Aharonov-Bohm oscillations or universal conductance fluctuations. In the currently accepted picture for the FQHE [30], the fractional states can be understood as the IQHE of composite fermions (an electron with two fictitious flux quanta attached). In a mean field approximation the composite fermions move in a zero effective magnetic field at filling factor $\nu=1 / 2$. However, fluctuations in the electron density give rise to fluctuations in the effective magnetic field which are sufficient to destroy time reversal symmetry and thus suppress weak localisation. Aharonov-Bohm oscillations do not require time reversal symmetry and as such should be more resistant to magnetic field fluctuations. Nevertheless, Aharonov-Bohm oscillations are not observed for composite fermions [31]. The observation of a low temperature phase for the fractional filling factors is therefore surprising and if interpreted as being due to quantum interference this would be to the best of our knowledge the first observation of a physical effect related to coherence for composite fermions. 


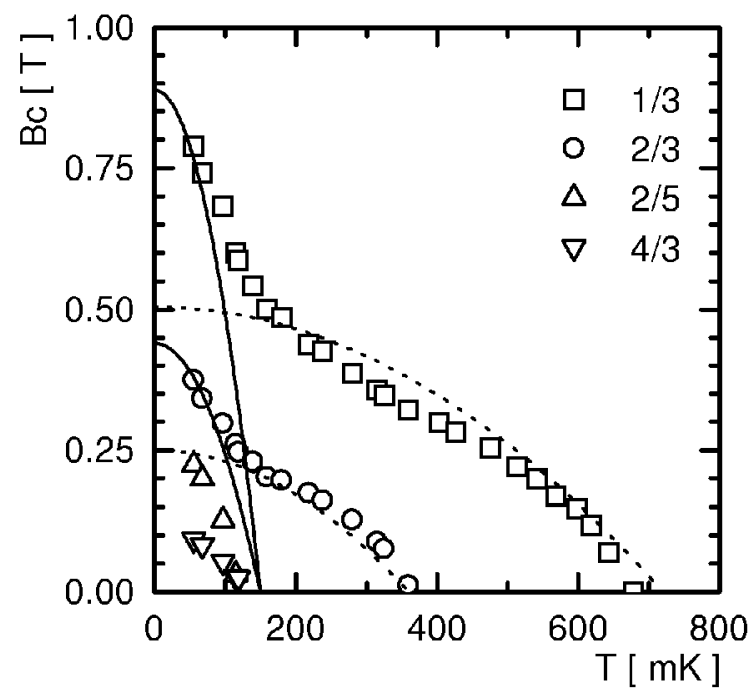

Fig. 8. Critical magnetic field $B_{\mathrm{c}}$ as a function of temperature for different fractions for the $n=1.6 \times 10^{11} \mathrm{~cm}^{-2}$ heterojunction sample. The dotted and dashed lines are guides to the eye generated using a Gorter-Casimir type expression.

\section{Discussion and conclusion}

The analogy between the dissipationless conductance in the fractional quantum Hall effect and superconductivity has been discussed in a number of theoretical papers [1-3]. Girvin and MacDonald [1] proposed an effective-field-theory model analogous to the Landau-Ginzburg theory of superconductivity in which the FQHE can be viewed as a superconducting state of composite bosons. Aronov and Mirlin [4] demonstrated that the low temperature conductivity of such an anion gas in the presence of impurity scattering remains finite but tends exponentially towards zero with decreasing temperature. A gauge transformation to form composite bosons and associated anion superconductivity would seem irrelevant for the single electron description which works so well for the IQHE. The similarity between the phase diagrams must have its origins in the similarity of the Hamiltonians: the vortex term in the Hamiltonian of a $2 \mathrm{D}$ superconductor is identical to that of the Coulomb gas in two dimensions [22].

In conclusion, the phase diagram for the breakdown of the IQHE is well described phenomenologically by the equations of the two-fluid model for superconductivity. The phase diagram for the critical current can be well fitted by the Gorter-Casimir two-fluid model for type I superconductors. The phase diagram for the critical magnetic field is more complicated and is strikingly similar to the phase diagram for the coercive field in type II superconductors. The observed "melting" behaviour for the critical magnetic field is intriguing and seems to be linked to dissipation induced by passing current through the sample. Recent elastic tunnelling measurements on high temperature superconductors clearly demonstrate 
that when a "dissipationless" measuring technique is used the melting behaviour is not observed [32, 33].

For even filling factors a remarkably simple scaling law between different filling factors is observed. This scaling law is modified for odd filling factors due to the many-body nature of the spin gap. All filling factors (even, odd, and fractional) show a low temperature phase which is due to increased localisation. This is interpreted within the framework of the scaling theory of the QHE as being due to quantum interference of coherent electrons. If this interpretation is also correct for the fractional filling factors this is possibly the first observation of coherence for composite fermions. The similarity between the $\mathrm{HT}_{\mathrm{c}}$ and QHE phase diagrams is a striking one. Clearly many questions remain to be answered.

\section{References}

[1] S.M. Girvin, A.H. MacDonald, Phys. Rev. Lett. 58, 1252 (1987).

[2] R.B. Laughlin, Phys. Rev. Lett. 60, 2677 (1988).

[3] S.C. Zhang, T.H. Hansson, S. Kivelson, Phys. Rev. Lett. 62, 82 (1989).

[4] A.G. Aronov, A.D. Mirlin, Phys. Lett. A 152, 371 (1991).

[5] Y. Imry, Physica B 152, 295 (1988).

[6] V. Srivastava, Physica B 212, 299 (1995).

[7] G. Ebert, K. von Klitzing, K. Ploog, G. Weimann, J. Phys. C, Solid State Phys. 16, $5441(1983)$.

[8] S. Kawaji, Semicond. Sci. Technol. 11, 1546 (1996) and references therein.

[9] M.E. Cage, R.F. Dziuba, B.F. Field, E.R. Williams, S.M. Girvin, A.C. Gossard, D.C. Tsui, R.J. Wagner, Phys. Rev. Lett. 51, 1374 (1983).

[10] P.M. Mensz, D.C. Tsui, Phys. Rev. B 40, 3919 (1989).

[11] N.Q. Balaban, U. Meirav, H. Shtrikman, Y. Levinson, Phys. Rev. Lett. 71, 1443 (1993); N.Q. Balaban, U. Meirav, H. Shtrikman, Phys. Rev. B 52, R5503 (1995).

[12] S. Komiyama, T. Takamasu, S. Hiyamizu, S. Sasa, Solid State Commun. 54, 479 (1985).

[13] O. Heinonen, P.L. Taylor, S. Girvin, Phys. Rev. B 30, 3016 (1984).

[14] L. Eaves, F.W. Sheard, Semicond. Sci. Technol. 1, L346 (1986).

[15] S.A. Trugman, Phys. Rev. B 27, 7539 (1983).

[16] K. Ishikawa, N. Maeda, K. Tadaki, Phys. Rev. B 54, 17819 (1996).

[17] V. Tsemekhman, K. Tsemekham, C. Wexler, J.H. Han, D.J. Thouless, Phys. Rev. B 55, R10201 (1997).

[18] L.B. Rigal, D.K. Maude, M. Potemski, J.C. Portal, L. Eaves, Z.R. Wasilewski, G. Hill, M.A. Pate, Phys. Rev. Lett. 82, 1249 (1999).

[19] C.J. Gorter, H.B.G. Casimir, Physica 1, 306 (1934).

[20] H.P. Wei, D.C. Tsui, M.A. Paalanen, A.M. Pruisken, Phys. Rev. Lett. 61, 1294 (1988). See also E. Chow, H.P. Wei, Phys. Rev. B 52, 13749 (1995). 
[21] A.M. Pruisken, Phys. Rev. Lett. 61, 12997 (1988).

[22] G. Blatter, M.V. Feigel'man, V.B. Geshkenbein, A.I. Larkin, V.M. Vinokur, Rev. Mod. Phys. 66, 1125 (1994) and references therein.

[23] J.M. Kosterlitz, D.J. Thouless, J. Phys. C 6, 1181 (1973).

[24] S. Martin, A.T. Fiory, R.M. Fleming, G.P. Espinosa, A.S. Cooper, Phys. Rev. Lett. 62, 677 (1989).

[25] J.C. Garland, Hu Jong Lee, Phys. Rev. B 36, 3636 (1987).

[26] M.M. Fogler, B.I. Shklovski, Phys. Rev. B 52, 17366 (1995).

[27] D.R. Leadley, R.J. Nicholas, J.J. Harris, C.T. Foxon, Phys. Rev. B 58, 13036 (1998).

[28] T. Ando, Y. Uemura, J. Phys. Soc. Japan 37, 1044 (1974).

[29] C. Callin, B.I. Halperin, Phys. Rev. B 31, 3635 (1985); Phys. Rev. B 30, 5655 (1984).

[30] J.K. Jain, Phys. Rev. Lett. 63, 199 (1989); J.K. Jain, Adv. Phys. 41, 105 (1992).

[31] A. Sachrajda, Y. Feng, A. Delage, P. Kelly, H. Carmona, P.C. Main, L. Eaves, J. Phys. C 8, 3019 (1996).

[32] P. Samuely, P. Szabo, T. Klein, A.G.M. Jansen, J. Marcus, C. Escribe-Filippini, P. Wyder, Europhys. Lett. 41, 207 (1988).

[33] P. Szabo, P. Samuely, A.G.M. Jansen, J. Marcus, P. Wyder, Phys. Rev. B 62, 3502 (2000). 